\title{
Cones, tri-Sasakian structures and superconformal invariance
}

\author{
G.W. Gibbons* and P. Rychenkova ${ }^{\dagger}$ \\ DAMTP, University of Cambridge, Silver Street \\ Cambridge, CB3 9EW, U.K.
}

September 17, 2018

DAMTP-1998-129

\begin{abstract}
In this note we show that rigid $N=2$ superconformal hypermultiplets must have target manifolds which are cones over tri-Sasakian metrics. We comment on the relation of this work to cone-branes and the AdS/CFT correspondence.
\end{abstract}

\section{Introduction}

There has recently been great interest in rigid conformally invariant supersymmetric field theories. In particular de Wit, Kleijn and Vandoren [1] have studied $N=2$ models containing hypermultiplets taking values in a hyperKähler manifold $\left(\mathcal{M}, g_{\mu \nu}, I_{a}{ }^{\mu}{ }_{\nu}\right)$, where $\mu, \nu=1, \ldots, 4 k=\operatorname{dim} \mathcal{M}$ and $a=$ $1,2,3$. They find the following necessary condition that the target manifold admits an infinitesimal dilatation invariance: $\left(\mathcal{M}, g_{\mu \nu}\right)$ admits a vector field $X^{\mu}$ such that

$$
X_{; \nu}^{\mu}=\delta_{\nu}^{\mu}
$$

In this note we point out that condition (11) implies (regardless of any hyperKähler condition) that $\mathcal{M}$ is a cone, $C(B)$, over a base manifold $B$, i.e. in coordinates $x^{\mu}=\left(r, x^{i}\right), i=1, \ldots, \operatorname{dim} \mathcal{M}-1$, the metric $g_{\mu \nu}$ is

$$
g_{\mu \nu} d x^{\mu} d x^{\nu}=d r^{2}+r^{2} h_{i j} d x^{i} d x^{j},
$$

\footnotetext{
*e-mail:gwg1@damtp.cam.ac.uk
}

†e-mail:pr201@damtp.cam.ac.uk 
where $h_{i j}$ is the metric on the base $B$ which depends only on $x^{i}$. Moreover, in these coordinates

$$
X=r \frac{\partial}{\partial r}
$$

and so the dilatation acts as

$$
\left(r, x^{i}\right) \rightarrow\left(\lambda r, x^{i}\right), \quad \lambda \in \mathbb{R}^{+}
$$

The differential operator $X=r \partial / \partial r$ is sometimes called the Eulerian vector field.

In the case that $\left(\mathcal{M}, g_{\mu \nu}\right)$ is a Ricci-flat Kähler, condition (1) implies that the vector field $X^{\mu}$ is holomorphic, that the base manifold $B$ carries a Sasakian structure and hence the metric $g_{\mu \nu}$ admits a holomorphic Killing vector field

$$
Y^{\mu}=I_{\nu}^{\mu} X^{\nu}
$$

where $I_{\nu}^{\mu}$ is the complex structure. Presumably this case arises in $N=1$ rigid superconformally invariant theories [9]. If $\left(\mathcal{M}, g_{\mu \nu}\right)$ is Ricci-flat then $B$ must be Einstein-Sasakian. We do not know whether rigid $N=1$ superconformal invariance implies that the metric should be Ricci-flat.

In the case that $\left(\mathcal{M}, g_{\mu \nu}\right)$ is hyperKähler, the base manifold $B$ admits a tri-Sasakian structure and the metric $g_{\mu \nu}$ also admits an $S U(2)$ action by isometries which permutes the complex structures $I_{1}, I_{2}$ and $I_{3}$. In this case the metric is necessarily Ricci-flat and the base manifold is necessarily Einstein.

The organisation of the article is as follows. In section 2 we study equation (1) in an arbitrary metric $g_{\mu \nu}$ and show that it leads to equation (2). In section 3 we assume $(\mathcal{M}, g)$ is Kähler. In section 4 we assume $(\mathcal{M}, g)$ is hyperKähler. In section 5 we discuss a general homothety which does not satisfy (1). Section 6 contains examples and in section 6 we discuss applications of the results. We find it remarkable how simply our main results follow from equation (1) and, although there are a number of discussions of cone geometries in the pure mathematics literature, we believe that our simple and direct treatment will be especially appealing to field theorists.

\section{Cones and Dilatations}

A manifold $\left(\mathcal{M}, g_{\mu \nu}\right)$ regardless of signature of $g_{\mu \nu}$ admits a conformal Killing vector field $X$ if and only if

$$
\mathcal{L}_{X} g_{\mu \nu}=\phi g_{\mu \nu}=X_{\mu ; \nu}+X_{\nu ; \mu}
$$


for some smooth function $\phi$. If $\phi$ is constant $X^{\mu}$ is said to generate a homothety. If $X^{\mu}$ is hypersurface orthogonal, i.e.

$$
X_{\mu}=\partial_{\mu} f \Longleftrightarrow X_{\mu ; \nu}=X_{\nu ; \mu}
$$

for some function $f$, we say that $(\mathcal{M}, g)$ admits as infinitesimal dilatation. Since equations (3) and (蛋) are equivalent to equation (11) this is the situation we are interested in. It follows that

$$
\nabla_{\mu} \nabla_{\nu} f=g_{\mu \nu}
$$

Moreover, defining

$$
V=g^{\mu \nu} \partial_{\mu} f \partial_{\nu} f=g_{\mu \nu} X^{\mu} X^{\nu}
$$

we have

$$
\partial_{\mu} V=2 \partial_{\mu} f
$$

We pick the arbitrary constant of integration such that

$$
V=2 f
$$

Now we pick $f$ as one of the coordinates and find the metric to be

$$
d s^{2}=\frac{d f^{2}}{2 f}+g_{i j}\left(x^{r}, f\right) d x^{i} d x^{j} .
$$

There is no cross term in the metric because $X^{\mu}$ is orthogonal to the surface $f=$ const.

Finally, we write out the equation

$$
\mathcal{L}_{X} g_{\mu \nu}=g_{\mu \nu, \lambda} X^{\lambda}+g_{\mu \lambda} X_{, \nu}^{\lambda}+g_{\nu \lambda} X_{, \mu}^{\lambda}=2 g_{\mu \nu}
$$

Using the fact that

$$
X^{\mu}=g^{\mu \nu} \partial_{\nu} f=2 f \delta_{0}^{\mu}
$$

and substituting (7) into (8), we obtain for the $(i, j)$ component

$$
f \frac{\partial g_{i j}}{\partial f}=g_{i j}
$$

If we define

$$
r^{2}=2 f,
$$

the solution of (9) may be written as

$$
g_{i j}=r^{2} h_{i j}\left(x^{k}\right),
$$


and the basic result (2) follows.

In fact we need not assume that the metric $g_{\mu \nu}$ is Riemannian, but if $X^{\mu}$ were time-like we would need to adjust the signs in (2). Note that from (6) we cannot have $X^{\mu}$ light-like. From (5) we find that

$$
\nabla_{\lambda} \nabla_{\mu} \nabla_{\nu} f=0
$$

But $\left(\nabla_{\mu} \nabla_{\nu}-\nabla_{\nu} \nabla_{\mu}\right) X^{\alpha}=R_{\beta \mu \nu}^{\alpha} X^{\beta}$ gives

$$
R_{\beta \mu \nu}^{\alpha} X^{\beta}=0
$$

which contracted on $\alpha$ and $\mu$ gives

$$
X^{\beta} R_{\beta \nu}=0
$$

Obviously (10) is incompatible with $g_{\mu \nu}$ being an Einstein metric with non-vanishing scalar curvature. However, it is not incompatible with $g_{\mu \nu}$ being Ricci-flat, and indeed this will be true if the metric $h_{i j}$ on the base is Einstein such that

$$
R_{i j}=(n-1) h_{i j}
$$

where $n=\operatorname{dim} \mathcal{M}$. Such a metric $g_{\mu \nu}$ is called a Ricci-flat cone.

Note that the assumption (4) that the homothety $X^{\mu}$ is hypersurface orthogonal played an essential role. For example Chave, Tod and Valent [2] have exhibited a Ricci-flat (hyperKähler) four-metric which admits a homothety which is not hypersurface orthogonal.

We conclude this section by remarking that cones have arisen in supergravity theories under the guise of "generalized dimensional reduction." For example Pope et al [3] have used the scaling invariance of supergravity theories to construct solutions which are eleven-dimensional cones over ten-dimensional base manifolds.

\section{Kählerian cones and Sasakian structures}

Now we suppose that $\mathcal{M}=C(B)$ is a Kähler manifold with a (covariantly constant) complex structure $I$. We have

$$
\begin{aligned}
\mathcal{L}_{X} I_{\nu}^{\mu} & =I_{\nu ; \sigma}^{\mu} X^{\sigma}-I_{\nu}^{\sigma} X_{; \sigma}^{\nu}+I_{\sigma}^{\mu} X_{; \nu}^{\sigma} \\
& =-I_{\nu}^{\mu}+I_{\nu}^{\mu}=0 .
\end{aligned}
$$


Hence necessarily $X$ is a holomorphic vector field. The reader is cautioned however that since

$$
\mathcal{L}_{X} \omega=d\left(\iota_{X} \omega\right)+\iota_{X}(d \omega)=2 \omega
$$

where $\omega$ is the Kähler form with components $\omega_{\mu \nu}=g_{\mu \sigma} I_{\nu}^{\sigma}$, we have

$$
d\left(\iota_{X} \omega\right)=2 \omega
$$

and hence $X$ is not Hamiltonian and there is no conventional moment map.

The vector field

$$
K^{\mu}=I_{\nu}^{\mu} X^{\nu}
$$

satisfies

$$
K_{\mu ; \nu}=\omega_{\mu \nu}
$$

Thus $K$ is a Killing field and it is easily seen to be holomorphic and to commute with $X$. In addition, $K$ is a Hamiltonian vector field whose moment map is $f$ and hence the level sets of the moment map coincide with the base manifold $B . K^{\mu}$ is tangent to the base manifold $B$ and is therefore a Killing field of the metric $h_{i j}$. From (12) we have

$$
K_{\mu} K^{\mu}=X_{\nu} X^{\nu}=V
$$

Thus the length of the vector $K^{\mu}$ is constant along base manifold $B$. Choosing $V=1$ as our base manifold we have the following structure on $B$ :

- a one-form $\eta_{i}=K_{i}$

- a vector field $\xi^{i}=K^{i}$

- an endomorphism $I_{j}^{i}$ of the tangent bundle $T(B)$ of $B$

- a metric $h_{i j}$.

It is straightforward to check that $\left(B, \xi^{i}, \eta_{i}, I^{i}{ }_{j}\right)$ satisfies the conditions for a Sasakian manifold [4].

Note that we have not assumed that $M$ is Ricci-flat. If we did so, then $\left(B, h_{i j}\right)$ would necessarily be an Einstein-Sasakian manifold. 


\section{HyperKählerian cones and tri-Sasakian structures}

Now we suppose $\mathcal{M}=C(B)$ is hyperKähler and hence necessarily Ricci-flat. The base metric must therefore be Einstein. The vector field $X$ is tri-holomorphic, i.e. it preserves the three complex structures $I_{a}$ and their algebra. There are three Killing vector fields tangent to $B$ and commuting with $X$ :

$$
K_{a}^{\mu}=I_{a}{ }^{\mu}{ }_{\nu} X^{\nu}
$$

However now

$$
\mathcal{L}_{K_{a}} I_{b}=-2 \epsilon_{a b c} I_{c}
$$

and

$$
\left[K_{a}, K_{b}\right]=-2 \epsilon_{a b c} K_{c}
$$

Thus we have a non-triholomorphic $S U(2)$ action on $\mathcal{M}$ which again descends to the base manifold $B$. In fact we now have a tri-Sasakian structure on $B$. Each generator $K_{a}$ of the $S U(2)$ action is holomorphic with respect to its own complex structure $I_{a}$ and $f$ is the associated moment map. The emergence of an extra $S U(2)$ isometry group was noticed in [1]. For more information and references to the mathematical literature on tri-Sasakian structures the reader is directed to [5].

\section{Hypersurface non-orthogonality}

As we emphasised above the assumption (4) that the homothetic Killing field $X$ is hypersurface orthogonal is essential for our result. Suppose that $X$ is a homothety which is not hypersurface orthogonal. Defining

$$
F_{\mu \nu}=\partial_{\mu} X_{\nu}-\partial_{\nu} X_{\nu}
$$

one finds that

$$
\mathcal{L}_{X} I^{\mu}{ }_{\nu}=I^{\mu}{ }_{\sigma} F_{\nu}^{\sigma}-F^{\mu}{ }_{\sigma} I_{\nu}^{\sigma}
$$

Thus, in general, $X$ need not be holomorphic with respect to any complex structure. Moreover, defining $K$ as in (12) we have

$$
\mathcal{L}_{K} g_{\mu \nu}=\omega_{\mu \sigma} F_{\nu}^{\sigma}+\omega_{\nu \sigma} F_{\mu}^{\sigma}
$$

Therefore we do not necessarily have an extra isometry. One might wonder whether, assuming $\mathcal{M}$ is hyperKähler, any non-trivial homothety could exist. In their paper Chave et al [2] have given 
a family of four-dimensional hyperKähler metrics with a tri-holomorphic homothety which is not hypersurface orthogonal. The metric is of the form

$$
e^{2 t}\left\{\frac{1}{W}(d t+A)^{2}+W \tilde{h}_{i j} d x^{i} d x^{j}\right\}
$$

where the metric in the base gives a $(4,0)$ sigma model and $W$ and $A$ satisfy monopole-like equations.

\section{Symmetry enhancement and examples}

There is no shortage of examples of tri-Sasakian manifolds (see [5] and references therein.) However, unless we take $B$ to be a sphere $S^{4 k-1}$ with its standard tri-Sasakian structure, the manifold $\mathcal{M}$ will be singular at the vertex $r=0$. In some cases the singularity may be removed to give a nonsingular hyperKähler manifold which no longer admits an exact dilatation symmetry but continues to do so approximately at infinity.

The obvious example are the ALE cones for which the base $B$ is $S^{3} / \Gamma$, where $\Gamma$ is a finite subgroup $\Gamma \subset S U(2) \subset S O(4)$. They may be thought of as the quotient of $\mathbb{R}^{4}$ by $\Gamma$ with an orbifold fixed point at the origin. As is well known [6] this may be blown up to give a non-singular manifold. It is instructive to consider the multi-centre case (see e.g. [7]). This may be constructed [8] as the hyperKähler quotient

$$
\mathbb{H}^{m+1} / /(U(1))^{m}
$$

The level sets of the moment maps are

$$
\mu_{\alpha}=q_{\alpha} i \bar{q}_{\alpha}+q i \bar{q}=\zeta_{\alpha}
$$

where $\alpha=1, \ldots, m$ and the quaternions $\left(q_{\alpha}, q\right)$ parametrise $\mathbb{H}^{m+1}$. The quantities $\left(\zeta_{\alpha}-\zeta_{\beta}\right)$ correspond to the relative separation of the centres. Now let $\zeta_{\alpha} \rightarrow 0$ for all $\alpha$. We get the orbifold limit in which the size of all two-cycles shrinks to zero. In the same limit the level sets (13) become invariant under the dilatation of $\mathbb{H}^{m+1}$ given by

$$
\left(q_{\alpha}, q\right) \longrightarrow\left(\lambda q_{\alpha}, \lambda q\right)
$$

which descends to the quotient orbifold. Thus the appearance of the dilatation symmetry is associated with the shrinking of two-cycles. Note that a general ALE metric has no $S U(2)$ isometry, 
tri-holomorphic or not. As we approach the orbifold limit the isometry group is enhanced to include $\mathbb{R}_{+} \times S U(2)$ where $\mathbb{R}_{+}$corresponds to dilatations. Note also that although there are many hyperKähler manifolds with non-triholomorphic $S U(2)$ actions they are not all cones. Neither are they necessarily asymptotically conical. For example all BPS monopole moduli spaces admit such an $S U(2)$ or $S O(3)$ action which arises from rotations in physical space, but they do not admit dilatations because of the scale set by the monopole mass.

An interesting question for further study is whether one can construct non-locally flat dilatation invariant hyperKähler manifolds using the hyperKähler quotient construction of a flat space.

\section{Discussion}

Cones over Sasakian and tri-Sasakian manifolds have recently made an appearance in M-theory [5, 9]. One considers $p$-brane solutions of the form

$$
H^{-\alpha}\left(-d t^{2}+d \mathbf{x}_{p}^{2}\right)+H^{\frac{2}{\beta}} g_{C}
$$

with $H=1+(\alpha / r)^{\beta}$ and $g_{C}$ the metric on a Ricci-flat cone with base $B$. These interpolate between $\mathbb{E}^{p, 1} \times C(B)$ at infinity and $A d S_{p+2} \times B$ near the throat. This supergravity solution corresponds to a large number, $k$, of Dirichlet $p$-branes.

The general belief is that the $U(1)$ factor of the world-volume $U(k)$ gauge theory is associated with the centre of mass motion. The $(9-p)$ scalars give the transverse coordinates of the branes.

The amount of supersymmetry of the world-volume theory is expected to agree with amount of supersymmetry of the supergravity background.

If $p=3$ it is tempting to make a connection with the four-dimensional rigid $N=2$ conformally invariant theories considered in [1]. However although cones appear both in the construction of the bulk space-time and as the target space of the world-volume theory the cones are, in general, not the same. The base $B$ of the cone used to construct the bulk space-time is five-dimensional and Einstein-Sasakian. The base of the cone of the target space of a putative $N=2$ world-volume theory must be $(4 n-1)$-dimensional and tri-Sasakian. Moreover, the amounts of supersymmetry of the supergravity solution and the world-volume theory do not agree.

We have a better bet with $N=1$ superconformal theories based on six-dimensional CalabiYau cones. The idea would be that the six centre of mass coordinates of the three-branes should assemble into three complex Higgs fields of the world-volume theory. This appears to coincide with 
the example considered in [9]: one takes $B=(S U(2) \times S U(2)) / U(1)$ with its Einstein-Sasakian structure.

For the M2-brane the cone of the supergravity solution is seven-dimensional and this could be taken to be tri-Sasakian. One might then contemplate identifying a hypermultiplet of the $(2+1)$ dimensional world-volume theory with the coordinates transverse to the M2-brane. However this looks rather artificial and suggests that one should look elsewhere for the geometrical origin of the hypermultiplets. By analogy with our discussion for the D3-brane it would seem to be more fruitful to follow [9] and consider three-dimensional $N=2$ world-volume theoriest] associated to an eight-dimensional Calabi-Yau cone. The case analysed in 9 is $B=S O(5) / S O(3)$ with its standard Einstein-Sasakian structure.

\section{Acknowledgements}

GWG would like to thank B. de Wit and S. Vandoren for raising the question of the geometrical significance of the equation (1) during the Ecole d'èté at ENS in August 1998. He would also like to thank Eugene Cremmer and other members of the ENS for their hospitality. PR thanks Trinity College, University of Cambridge, for a studentship.

Our discussion in section 7 owes a great deal to the comments of S.Vandoren and J.FigueroaO'Farrill on an earlier draft of this paper. We also thank J.Figueroa-O'Farrill for drawing our attention to an earlier paper by K.Galicki [10] who considered locally supersymmetric $N=2$ theories, rather than rigid superconformal theories considered in [1], and related them to cones. However he did not explicitly derived equation (11) and its consequences.

\section{References}

[1] B. de Wit, B. Kleijn and S. Vandoren, Rigid $N=2$ superconformal hypermultiplets, hepth/9808160.

[2] T. Chave, K. Tod and G. Valent, $(4,0)$ and $(4,4)$ sigma models with tri-holomorphic Killing vector, Phys.Lett. B383 (1996) 262.

[3] I. Lavrinenko, H. Lü and C. Pope, Fibre bundles and generalized dimensional reductions, Class.Quant.Grav. 15 (1998) 2239, hep-th/9710243.

\footnotetext{
${ }^{1}$ The counting $N=2$ is from the three-dimensional point of view.
} 
[4] D. Blair, Contact Manifolds in Riemannian Geometry, Lecture Notes in Math. 509 SpringerVerlag, 1976.

[5] B. Acharya, J. Figueroa-O'Farrill, C. Hull and B. Spence, Branes at conical singularities and holography, hep-th/9808014.

[6] P. Kronheimer, The construction of ALE spaces as hyperKähler quotients, J.Diff.Geom. 29 (1989) 665.

[7] G. Gibbons and S. Hawking, Gravitational multi-instantons, Phys. Lett. B78(4) (1978) 430.

[8] G.W.Gibbons, P.Rychenkova and R.Goto, HyperKähler quotient construction of BPS monopole moduli spaces, Comm.Math.Phys. 186 (1997) 581, hep-th/9608085.

[9] I. Klebanov and E. Witten, Superconformal field theory on threebranes at a Calabi-Yau singularity, hep-th/9807080.

[10] K. Galicki, Geometry of the scalar couplings in $N=2$ supergravity models, Class.Quan.Grav. 9(1) (1992) 27. 